\title{
Regulation of Tuberoinfundibular Dopamine (TIDA) Neurons by Kisspeptin Neurons
}

\author{
Hitoshi Ozawa ${ }^{1}$, Nobuhiko Sawai ${ }^{1,2}$, Kinuyo Iwata ${ }^{1}$, \\ Ken Takumi ${ }^{1}$ and Norio Iijima ${ }^{1}$ \\ ${ }^{1}$ Department of Anatomy and Neurobiology, Graduate School of Medicine, Nippon Medical School \\ ${ }^{2}$ Department of Anatomy and Cell Biology, Graduate School of Medicine, Gunma University
}

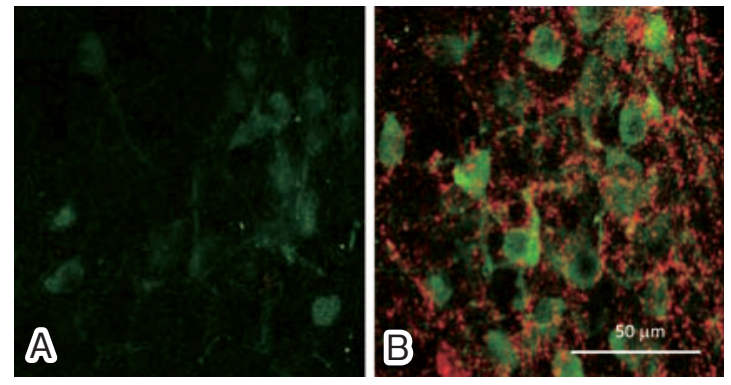

Fig. 1

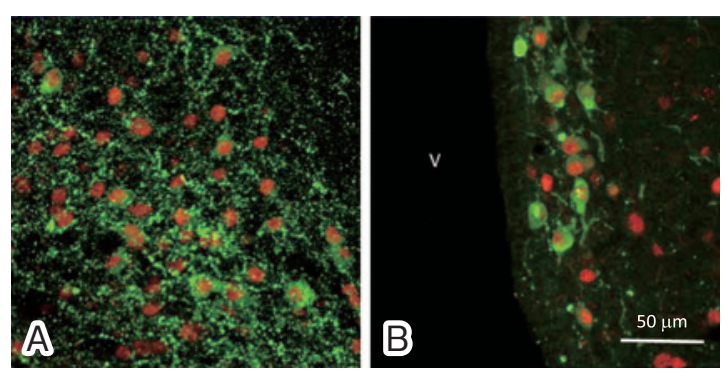

Fig. 3
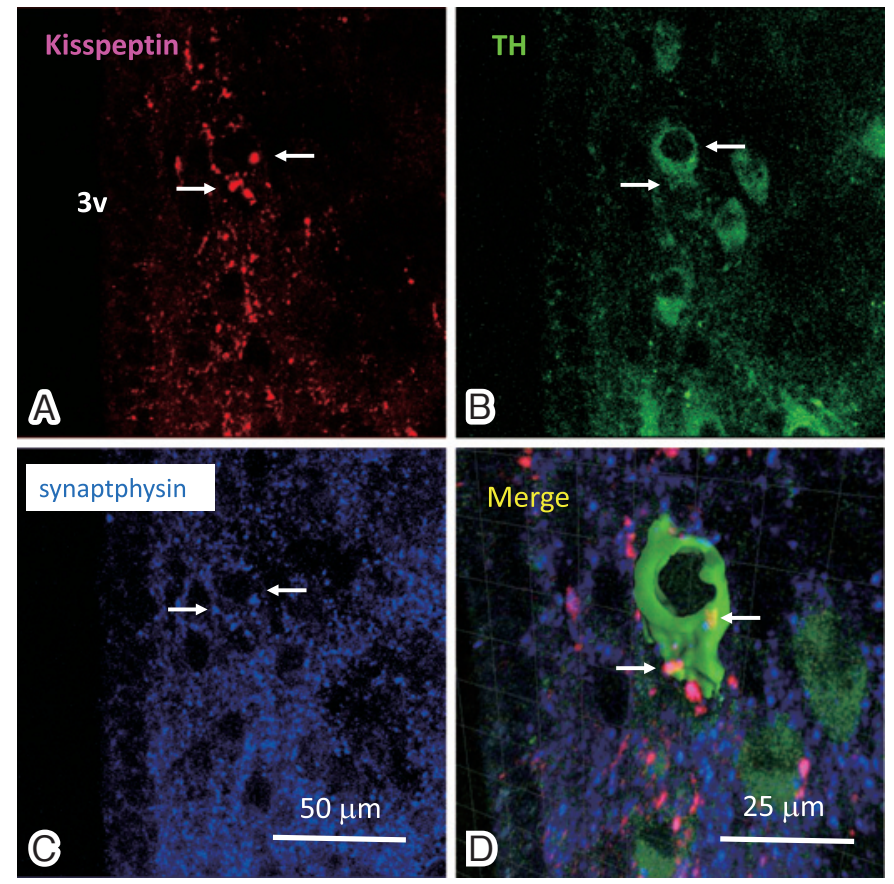

Fig. 2

\section{Introduction}

A new bioactive neuropeptide, "kisspeptin", the product of Kiss1 gene acting via GPR54 (also Kiss1 R) has recently been thought as an important gatekeeper of puberty onset and reproduction ${ }^{1,2}$. Much of recent focus on the regulation of gonadotropin-releasing hormone $(\mathrm{GnRH})$ secretion has been upon kisspeptin neurons located in the preoptic area (POA) and hypothalamus ${ }^{3,4}$. Many studies suggest that kisspeptin plays a key role in mediating the feedback effects of gonadal steroid hormones on GnRH neuroactivity during puberty, the estrous cycle, and/ or seasonal reproductive transitions ${ }^{1,2.5}$. The distribution of kisspeptin neurons has been identified by immunohistochemistry and in situ hybridization. In rodents, two distinguished populations have been reported: one is at the anteroventral periventricular (AVPV) nucleus which received positive feedback effect of sex steroids; the other located at the arcuate (ARC) nucleus which received negative feedback effect of sex steroids.

Recently, we have found the interesting projection of kisspeptin neurons to the tuberoinfundibular dopamine (TIDA) neurons, which are thought to inhibit prolactin secretion of mammotrohps in the anterior pituitary.

Here, we demonstrate interesting images indicating the neuronal communication between kisspeptin and TIDA neurons in the rat hypothalamus from recent our studies.

Fig. 1 The double immunofluorescent micrographs of kisspeptin (red) and tyrosine hydroxidase (TH) (green) in the dorsal hypothalamic area of the male (A) and the female (B) rat. The THimmunopositive cell bodies surrounded by the kisspeptin-immunoreactive fibers are preferentially observed in female rats. Bar $=50 \mu \mathrm{m}$

Correspondence to Hitoshi Ozawa, Department of Anatomy and Neurobiology, Graduate School of Medicine, Nippon

Medical School, 1-1-5 Sendagi, Bunkyo-ku, Tokyo 113-8602, Japan 


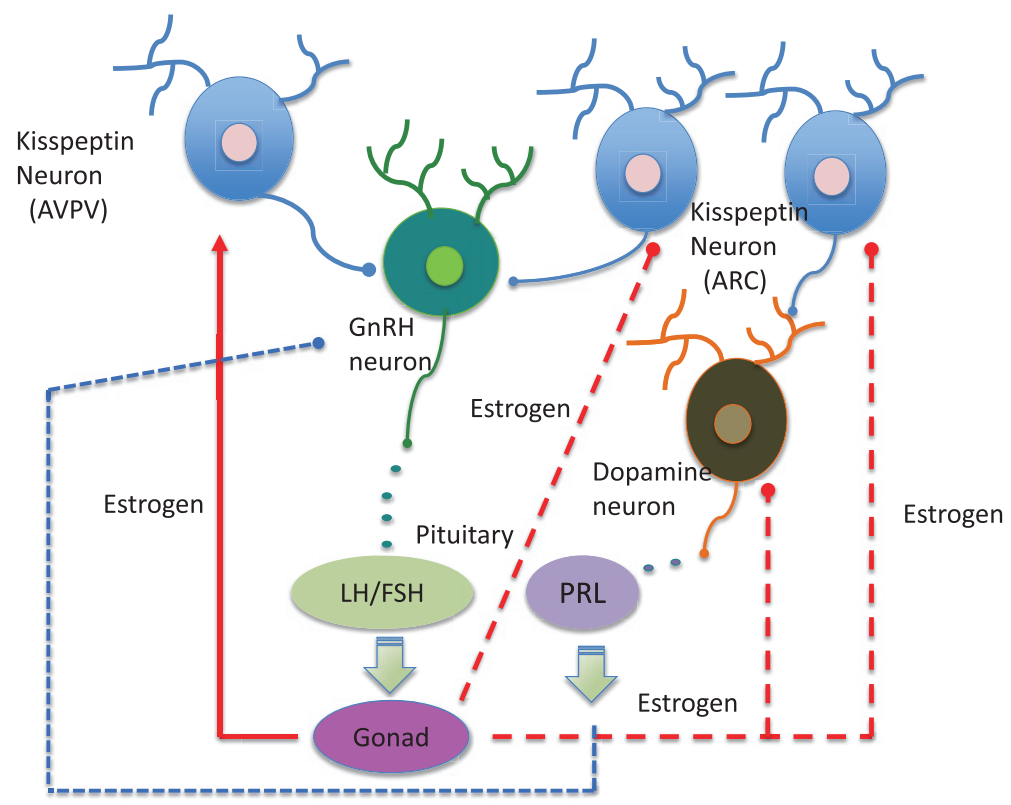

Fig. 4

Fig. 2 Triple immunofluorescent micrographs of kisspeptin (red) (A), TH (green) (B), and synaptophysin (blue) (C), a marker for the pre-synapse showed that these immunoreactions are expressed on the same cell body of neurons (white arrows). The merge microphotograph (D) which is threedimentionalized by a computer software, IMARIS, clearly indicates the co-localization of kisspeptin, $\mathrm{TH}$, and synaptophysin on the cell body of a TH-immuoreactive neuron in the dorsal hypothalamus of female rat, indicating that the kisspeptin neurons innervate the tubuloinfundibular dopamine (TIDA) neurons labeled by $\mathrm{TH}$ in the dorsal hypothalamus via synaptic contact. Bar $=50 \mu \mathrm{m}(\mathrm{A}, \mathrm{B}$, C) and $25 \mu \mathrm{m}(\mathrm{D})$

Fig. 3 Double immunofluorescent micrographs of estrogen receptor alpha (ER $\alpha)$ (red) and kisspeptin (green) in the arcuate nucleus (A), and of estrogen receptor alpha (ER $\alpha$ ) (red) and THimmunopositive TIDA neurons (green) in the dorsal hypothalamic area in the rat, indicating that both kisspeptin neurons and TIDA neurons are directly regulated by estrogens, a sex steroid of female, via their receptors. Bar $=50 \mu \mathrm{m}$

Fig. 4 Schematic summarizing of the communication of kisspeptin neurons in the rat hypothalamus. Kisspeptin neurons project to the GnRH neurons and regulate the hypothalamus-pituitary-gonad (HPG) axis. The kisspeptin neurons receive the feedback of HPG axis by estrogens via their receptors. Additionally, kisspeptin neurons also innervate the TIDA neurons, which are well known as an inhibitory regulator for prolactin secretion in the anterior pituitary. The TIDA neurons are also mediated by estrogens via their receptors, as well as kisspeptin neurons. (The solid line means the positive feedback, while the dotted line the negative feedback.)

\section{Conclusion}

Together with recent findings of the co-expression of neurokinin B (NKB) and dynorphin in the kisspeptin neurons in the arcuate nucleus ${ }^{2}$, kisspeptin/NKB/dynorphin neurons may directory affect TIDA neurons that regulate prolactin secretion, which is also related to the $\mathrm{GnRH}$ secretion in the hypothalamus.

\section{References}

1. Tena-Sempere M: Kisspeptin/GPR54 system as potential target for endocrine disruption of reproductive development and function. Internatinal Journal of Andrology 2010; 33: 360-368.

2. Lehman MN, Coolen LM, Goodman RL: Kisspeptin/Neurokinin B/Dynorphin (KNDy) cells of the arcuate nucleus: A central node in the control of gonadotropin-releasing hormone secretion. Endocrinology 2010; 151: 3479-3489.

3. Takumi K, Iijima N, Ozawa H: Developmental changes in the expression of kisspeptin mRNA in rat hypothalamus. Journal of Molecular Neruoscience 2011; 43: 138-145.

4. Iijima N, Takumi K, Sawai N, Ozawa H: An immunohistochemical study on the exressional dynamics of kisspeptin neurons relevant to GnRH neurons using a newly developed anti-kisspeptin antibody. Journal of Molecular Neruoscience 2011; 43: 146-154.

5. Ozawa H: Steroid hormones, their receptors and neuroendocrine system. J Nippon Med Sch 2005; 72: 316-325.

E-mail: hozawa@nms.ac.jpＪournal Website (http://www.nms.ac.jp/jnms/) 